\title{
EXISTENCE AND UNIQUENESS OF SOLUTIONS \\ FOR A CLASS OF INFINITE-HORIZON \\ SYSTEMS DERIVED FROM OPTIMAL CONTROL
}

LIANWEN WANG

Received 4 May 2004 and in revised form 14 February 2005

This paper deals with the existence and uniqueness of solutions for a class of infinitehorizon systems derived from optimal control. An existence and uniqueness theorem is proved for such Hamiltonian systems under some natural assumptions.

\section{Introduction}

We begin with a simple example to introduce the background of the considered problem. Let $U$ be a bounded closed subset of $\mathbb{R}^{m}$ and let functions $f: \mathbb{R}^{n} \times \mathbb{R}^{m} \times[a, \infty) \rightarrow \mathbb{R}^{n}$, $L: \mathbb{R}^{n} \times \mathbb{R}^{m} \times[a, \infty) \rightarrow \mathbb{R}$ be differentiable with respect to the first variable. Consider an optimal control system of the form

$$
\text { Minimize } J[u(\cdot)]=\int_{a}^{\infty} L(x(t), u(t), t) d t
$$

over all admissible controls $u(\cdot) \in L^{2}([a, \infty) ; U)$, where the trajectories $x:[a, \infty) \rightarrow \mathbb{R}^{n}$ are differentiable on $[a, \infty)$ and satisfy the dynamic system

$$
\dot{x}(t)=f(x(t), u(t), t), \quad x(a)=x_{0} .
$$

From control theory, the well-known Pontryagin maximum principle, an important necessary optimality condition, is usually applied to get optimal controls for this system. By doing this, the following infinite-horizon Hamiltonian system is derived:

$$
\begin{gathered}
\dot{x}(t)=\frac{\partial H(x(t), p(t), t)}{\partial p}, \\
\dot{x}(a)=x_{0}, \\
\dot{p}(t)=\frac{-\partial H(x(t), p(t), t)}{\partial x}, \\
x(\cdot) \in L^{2}\left([a, \infty) ; \mathbb{R}^{n}\right), \quad p(\cdot) \in L^{2}\left([a, \infty) ; \mathbb{R}^{n}\right) .
\end{gathered}
$$


Here, $H(x, p, t)=\lambda L(x, \bar{u}, t)+\langle p, f(x, \bar{u}, t)\rangle$ is the Hamiltonian function for (1.1)-(1.2), $\langle\cdot, \cdot\rangle$ stands for inner product in $\mathbb{R}^{n}, \bar{u}$ is an optimal control, and $x(t)$ is the optimal trajectory corresponding to the optimal control $\bar{u}$.

The existence and uniqueness of solutions for system (1.3) is a very interesting question; if solutions to (1.3) are unique, then the optimal control for system (1.1)-(1.2) can be solved analytically or numerically through (1.3). When we consider the generalization of (1.3) in infinite-dimensional spaces, the following Hamiltonian system is obtained:

$$
\begin{gathered}
\dot{x}(t)=A(t) x(t)+F(x(t), p(t), t), \\
x(a)=x_{0}, \\
\dot{p}(t)=-A^{*}(t) p(t)+G(x(t), p(t), t), \\
x(\cdot) \in L^{2}([a, \infty) ; X), \quad p(\cdot) \in L^{2}([a, \infty) ; X),
\end{gathered}
$$

where both $x(t)$ and $p(t)$ take values in a Hilbert space $X$ for $a \leq t<\infty$. It is always assumed that $F, G: X \times X \times[a, \infty) \rightarrow X$ are nonlinear operators, that $A(t)$ is a closed operator for each $t \in[a, \infty)$, and that $A^{*}(t)$ is the adjoint operator of $A(t)$.

The following system is called a linear Hamiltonian system, which is a special case of (1.4),

$$
\begin{gathered}
\dot{x}(t)=A(t) x(t)+B(t) p(t)+\varphi(t), \\
x(a)=x_{0}, \\
\dot{p}(t)=-A^{*}(t) p(t)+C(t) x(t)+\psi(t), \\
x(\cdot) \in L^{2}([a, \infty) ; X), \quad p(\cdot) \in L^{2}([a, \infty) ; X),
\end{gathered}
$$

where $\varphi(\cdot), \psi(\cdot) \in L^{2}([a, \infty) ; X)$, and $B(t), C(t)$ are selfadjoint linear operators from $X$ to $X$ for all $t \in[a, \infty)$.

In [2], Lions has discussed the existence and uniqueness of solutions for system (1.5) and gave an existence and uniqueness result. In [1], Hu and Peng considered the existence and uniqueness of solutions for a class of nonlinear forward-backward stochastic differential equations similar to (1.3) but on finite horizon, they provided an existence and uniqueness theorem for (1.3). Peng and Shi in [3] dealt with the existence and uniqueness of solutions for (1.3) using the techniques developed in [1]. In this paper, we consider the existence and uniqueness of solutions for infinite-dimensional system (1.4).

Throughout the paper, the following basic assumptions hold.

(I) There exists a real number $L>0$ such that

$$
\begin{gathered}
\left\|F\left(x_{1}, p_{1}, t\right)-F\left(x_{2}, p_{2}, t\right)\right\| \leq L\left(\left\|x_{1}-x_{2}\right\|+\left\|p_{1}-p_{2}\right\|\right), \\
\left\|G\left(x_{1}, p_{1}, t\right)-G\left(x_{2}, p_{2}, t\right)\right\| \leq L\left(\left\|x_{1}-x_{2}\right\|+\left\|p_{1}-p_{2}\right\|\right)
\end{gathered}
$$

for all $x_{1}, p_{1}, x_{2}, p_{2} \in X$ and $t \in[a, \infty)$. 
(II) There exists a real number $\alpha>0$ such that

$$
\begin{aligned}
& \left\langle F\left(x_{1}, p_{1}, t\right)-F\left(x_{2}, p_{2}, t\right), p_{1}-p_{2}\right\rangle+\left\langle G\left(x_{1}, p_{1}, t\right)-G\left(x_{2}, p_{2}, t\right), x_{1}-x_{2}\right\rangle \\
& \quad \leq-\alpha\left(\left\|x_{1}-x_{2}\right\|+\left\|p_{1}-p_{2}\right\|\right)
\end{aligned}
$$

for all $x_{1}, p_{1}, x_{2}, p_{2} \in X$ and $t \in[a, \infty)$.

\section{Lemmas}

Two lemmas are given in this section. They are essential to prove the main theorem.

Lemma 2.1. Consider the Hamiltonian system

$$
\begin{gathered}
\dot{x}(t)=A(t) x(t)+F_{\beta}(x, p, t)+\varphi(t), \\
x(a)=x_{0}, \\
\dot{p}(t)=-A^{*}(t) p(t)+G_{\beta}(x, p, t)+\psi(t), \\
x(\cdot) \in L^{2}([a, \infty) ; X), \quad p(\cdot) \in L^{2}([a, \infty) ; X),
\end{gathered}
$$

where $\varphi(\cdot), \psi(\cdot) \in L^{2}([a, \infty) ; X)$. The functions $F_{\beta}$ and $G_{\beta}$ are defined as

$$
\begin{aligned}
& F_{\beta}(x, p, t):=-(1-\beta) \alpha p+\beta F(x, p, t), \\
& G_{\beta}(x, p, t):=-(1-\beta) \alpha x+\beta G(x, p, t)
\end{aligned}
$$

Assume that (2.1) has a unique solution for some real number $\beta=\beta_{0} \geq 0$ and any $\varphi(t), \psi(t)$. There exists a real number $\delta>0$, which is independent of $\beta_{0}$, such that (2.1) has a unique solution for any $\varphi(t), \psi(t)$, and $\beta \in\left[\beta_{0}, \beta_{0}+\delta\right]$.

Proof. For any given $\varphi(\cdot), \psi(\cdot), x(\cdot), p(\cdot) \in L^{2}([a, \infty) ; X)$ and $\delta>0$, construct the following Hamiltonian system:

$$
\begin{gathered}
\dot{X}(t)=A(t) X(t)+F_{\beta_{0}}(X, P, t)+F_{\beta_{0}+\delta}(x, p, t)-F_{\beta_{0}}(x, p, t)+\varphi(t), \\
X(a)=x_{0}, \\
\dot{P}(t)=-A^{*}(t) P(t)+G_{\beta_{0}}(X, P, t)+G_{\beta_{0}+\delta}(x, p, t)-G_{\beta_{0}}(x, p, t)+\psi(t), \\
X(\cdot) \in L^{2}([a, \infty) ; X), \quad P(\cdot) \in L^{2}([a, \infty) ; X) .
\end{gathered}
$$

Note that

$$
\begin{aligned}
& F_{\beta_{0}+\delta}(x, p, t)-F_{\beta_{0}}(x, p, t) \\
& \quad=-\left(1-\beta_{0}-\delta\right) \alpha p+\left(\beta_{0}+\delta\right) F(x, p, t)+\left(1-\beta_{0}\right) \alpha p-\beta_{0} F(x, p, t) \\
& \quad=\alpha \delta p+\delta F(x, p, t), \\
& G_{\beta_{0}+\delta}(x, p, t)-G_{\beta_{0}}(x, p, t) \\
& \quad=-\left(1-\beta_{0}-\delta\right) \alpha x+\left(\beta_{0}+\delta\right) G(x, p, t)+\left(1-\beta_{0}\right) \alpha x-\beta_{0} G(x, p, t) \\
& \quad=\alpha \delta x+\delta G(x, p, t) .
\end{aligned}
$$


The assumption of Lemma 2.1 implies that (2.3) has a unique solution for each pair $(x(\cdot), p(\cdot)) \in L^{2}([a, \infty) ; X) \times L^{2}([a, \infty) ; X)$. Therefore, the mapping $J$,

$$
L^{2}([a, \infty) ; X) \times L^{2}([a, \infty) ; X) \longrightarrow L^{2}([a, \infty) ; X) \times L^{2}([a, \infty) ; X),
$$

given by

$$
J(x(\cdot), p(\cdot)):=(X(\cdot), P(\cdot))
$$

is well defined.

Let $J\left(x_{1}(\cdot), p_{1}(\cdot)\right)=\left(X_{1}(\cdot), P_{1}(\cdot)\right)$ and $J\left(x_{2}(\cdot), p_{2}(\cdot)\right)=\left(X_{2}(\cdot), P_{2}(\cdot)\right)$. Since $X_{1}(\cdot)-$ $X_{2}(\cdot) \in L^{2}([a, \infty) ; X)$ and $P_{1}(\cdot)-P_{2}(\cdot) \in L^{2}([a, \infty) ; X)$, there exists a sequence of real numbers $a<t_{1}<t_{2}<\cdots<t_{k}<\cdots$ such that $t_{k} \rightarrow \infty$ as $k \rightarrow \infty$ and

$$
X_{1}\left(t_{k}\right)-X_{2}\left(t_{k}\right) \longrightarrow 0, \quad P_{1}\left(t_{k}\right)-P_{2}\left(t_{k}\right) \longrightarrow 0, \text { as } k \longrightarrow \infty \text {. }
$$

Note that

$$
\begin{aligned}
& \frac{d}{d t}\left\langle X_{1}(t)-X_{2}(t), P_{1}(t)-P_{2}(t)\right\rangle \\
& =\left\langle F_{\beta_{0}}\left(X_{1}, P_{1}, t\right)-F_{\beta_{0}}\left(X_{2}, P_{2}, t\right)+\alpha \delta\left(p_{1}-p_{2}\right)+\delta\left(F\left(x_{1}, p_{1}, t\right)-F\left(x_{2}, p_{2}, t\right)\right), P_{1}-P_{2}\right\rangle \\
& \quad+\left\langle G_{\beta_{0}}\left(X_{1}, P_{1}, t\right)-G_{\beta_{0}}\left(X_{2}, P_{2}, t\right)+\alpha \delta\left(x_{1}-x_{2}\right)+\delta\left(G\left(x_{1}, p_{1}, t\right)-G\left(x_{2}, p_{2}, t\right)\right), X_{1}-X_{2}\right\rangle \\
& :=I_{1}+I_{2} .
\end{aligned}
$$

Since

$$
F_{\beta_{0}}\left(X_{1}, P_{1}, t\right)-F_{\beta_{0}}\left(X_{2}, P_{2}, t\right)=-\alpha\left(1-\beta_{0}\right)\left(P_{1}-P_{2}\right)+\beta_{0}\left(F\left(X_{1}, P_{1}, t\right)-F\left(X_{2}, P_{2}, t\right)\right)
$$

implies that

$$
\begin{aligned}
I_{1}= & -\alpha\left(1-\beta_{0}\right)\left\|P_{1}-P_{2}\right\|^{2}+\beta_{0}\left\langle F\left(X_{1}, P_{1}, t\right)-F\left(X_{2}, P_{2}, t\right), P_{1}-P_{2}\right\rangle \\
& +\alpha \delta\left\langle p_{1}-p_{2}, P_{1}-P_{2}\right\rangle+\delta\left\langle F\left(x_{1}, p_{1}, t\right)-F\left(x_{2}, p_{2}, t\right), P_{1}-P_{2}\right\rangle,
\end{aligned}
$$

similarly,

$$
G_{\beta_{0}}\left(X_{1}, P_{1}, t\right)-G_{\beta_{0}}\left(X_{2}, P_{2}, t\right)=-\alpha\left(1-\beta_{0}\right)\left(X_{1}-X_{2}\right)+\beta_{0}\left(G\left(X_{1}, P_{1}, t\right)-G\left(X_{2}, P_{2}, t\right)\right)
$$

implies that

$$
\begin{aligned}
I_{2}= & -\alpha\left(1-\beta_{0}\right)\left\|X_{1}-X_{2}\right\|^{2}+\beta_{0}\left\langle G\left(X_{1}, P_{1}, t\right)-G\left(X_{2}, P_{2}, t\right), X_{1}-X_{2}\right\rangle \\
& +\alpha \delta\left\langle x_{1}-x_{2}, X_{1}-X_{2}\right\rangle+\delta\left\langle G\left(x_{1}, p_{1}, t\right)-G\left(x_{2}, p_{2}, t\right), X_{1}-X_{2}\right\rangle .
\end{aligned}
$$


It follows from the estimates for $I_{1}, I_{2}$, and the assumption (I) that

$$
\begin{aligned}
I_{1}+I_{2} \leq & -\alpha\left(\left\|X_{1}-X_{2}\right\|^{2}+\left\|P_{1}-P_{2}\right\|^{2}\right) \\
& +\alpha \delta\left(\left\|p_{1}-p_{2}\right\|\left\|P_{1}-P_{2}\right\|+\left\|x_{1}-x_{2}\right\|\left\|X_{1}-X_{2}\right\|\right) \\
& +\delta\left\|F\left(x_{1}, p_{1}, t\right)-F\left(x_{2}, p_{2}, t\right)\right\|\left\|P_{1}-P_{2}\right\| \\
& +\delta\left\|G\left(x_{1}, p_{1}, t\right)-G\left(x_{2}, p_{2}, t\right)\right\|\left\|X_{1}-X_{2}\right\| \\
\leq & -\alpha\left(\left\|X_{1}-X_{2}\right\|^{2}+\left\|P_{1}-P_{2}\right\|^{2}\right) \\
& +\delta(2 L+\alpha)\left(\left\|X_{1}-X_{2}\right\|^{2}+\left\|P_{1}-P_{2}\right\|^{2}+\left\|x_{1}-x_{2}\right\|^{2}+\left\|p_{1}-p_{2}\right\|^{2}\right) .
\end{aligned}
$$

Therefore,

$$
\begin{aligned}
\frac{d}{d t}\left\langle X_{1}(t)-X_{2}(t), P_{1}(t)-P_{2}(t)\right\rangle \\
\leq-\alpha\left(\left\|X_{1}-X_{2}\right\|^{2}+\left\|P_{1}-P_{2}\right\|^{2}\right) \\
\quad+\delta(2 L+\alpha)\left(\left\|X_{1}-X_{2}\right\|^{2}+\left\|P_{1}-P_{2}\right\|^{2}+\left\|x_{1}-x_{2}\right\|^{2}+\left\|p_{1}-p_{2}\right\|^{2}\right) .
\end{aligned}
$$

Integrating between $a$ and $t_{k}$, we have

$$
\begin{aligned}
\left\langle X_{1}\left(t_{k}\right)\right. & \left.-X_{2}\left(t_{k}\right), P_{1}\left(t_{k}\right)-P_{2}\left(t_{k}\right)\right\rangle-\left\langle X_{1}(a)-X_{2}(a), P_{1}(a)-P_{2}(a)\right\rangle \\
\leq & -\alpha \int_{a}^{t_{k}}\left(\left\|X_{1}-X_{2}\right\|^{2}+\left\|P_{1}-P_{2}\right\|^{2}\right) d t+\delta(2 L+\alpha) \\
& \times \int_{a}^{t_{k}}\left(\left\|X_{1}-X_{2}\right\|^{2}+\left\|P_{1}-P_{2}\right\|^{2}+\left\|x_{1}-x_{2}\right\|^{2}+\left\|p_{1}-p_{2}\right\|^{2}\right) d t .
\end{aligned}
$$

Letting $k \rightarrow \infty$ and noting that (2.7), we obtain

$$
\int_{a}^{\infty}\left(\left\|X_{1}-X_{2}\right\|^{2}+\left\|P_{1}-P_{2}\right\|^{2}\right) d t \leq \frac{2 \delta L+\delta \alpha}{\alpha-2 \delta L-\delta \alpha} \int_{a}^{\infty}\left(\left\|x_{1}-x_{2}\right\|^{2}+\left\|p_{1}-p_{2}\right\|^{2}\right) d t .
$$

Choose a small $\delta$ (independent of $\beta_{0}$ ) such that

$$
\frac{2 \delta L+\delta \alpha}{\alpha-2 \delta L-\delta \alpha} \leq \frac{1}{2}
$$

then $J$ is a contractive mapping and hence has a unique fixed point. Thus, (2.3) becomes

$$
\begin{gathered}
\dot{x}(t)=A(t) x(t)+F_{\beta_{0}+\delta}(x, p, t)+\varphi(t), \\
x(a)=x_{0}, \\
\dot{p}(t)=-A^{*}(t) p(t)+G_{\beta_{0}+\delta}(x, p, t)+\psi(t), \\
x(\cdot) \in L^{2}([a, \infty) ; X), \quad p(\cdot) \in L^{2}([a, \infty) ; X) .
\end{gathered}
$$

This shows that system (2.1) has a unique solution on $[a, \infty)$ for $\beta \in\left[\beta_{0}, \beta_{0}+\delta\right]$. The proof is complete. 
Lemma 2.2. System (2.1) has a unique solution on $[a, \infty)$ for $\beta=0$, that is, the system

$$
\begin{gathered}
\dot{x}(t)=A(t) x(t)-\alpha p(t)+\varphi(t), \\
x(0)=x_{0}, \\
\dot{p}(t)=-A^{*}(t) p(t)-\alpha x(t)+\psi(t), \\
x(\cdot) \in L^{2}([a, \infty) ; X), \quad p(\cdot) \in L^{2}([a, \infty) ; X),
\end{gathered}
$$

has a unique solution on $[a, \infty)$.

For the proof, see [2, Section 6.2, Chapter III].

\section{Main theorem}

Theorem 3.1. System (1.4) has a unique solution under assumptions (I) and (II).

Proof. By Lemma 2.2, system (2.1) has a unique solution on $[a, \infty)$ in the case $\beta_{0}=0$. It follows from Lemma 2.1 that there exists a real number $\delta>0$ such that (2.1) has a unique solution on $[a, \infty)$ for any $\beta \in[0, \delta]$ and $\varphi, \psi \in L^{2}([a, \infty) ; X)$. Let $\beta_{0}=\delta$ in Lemma 2.1. Repeating this procedure implies that $(2.1)$ has a unique solution on $[a, \infty)$ for any $\beta \in$ $[\delta, 2 \delta]$ and $\varphi, \psi \in L^{2}([a, \infty) ; X)$. After finitely many steps, one can show that system $(2.1)$ has a unique solution for $\beta=1$. Therefore, it is proved that system (1.4) has a unique solution on $[a, \infty)$ by letting $\beta=1, \varphi(t) \equiv 0$, and $\psi(t) \equiv 0$.

Remark 3.2. Consider system (1.5). Note that

$$
\begin{gathered}
\left\langle F\left(x_{1}, p_{1}, t\right)-F\left(x_{2}, p_{2}, t\right), p_{1}-p_{2}\right\rangle+\left\langle G\left(x_{1}, p_{1}, t\right)-G\left(x_{2}, p_{2}, t\right), x_{1}-x_{2}\right\rangle \\
=\left\langle B(t)\left(p_{1}-p_{2}\right), p_{1}-p_{2}\right\rangle+\left\langle C(t)\left(x_{1}-x_{2}\right), x_{1}-x_{2}\right\rangle .
\end{gathered}
$$

By Theorem 3.1, system (1.5) has a unique solution if it is assumed that both $B(t)$ and $C(t)$ are uniformly negative definite on $[a, \infty)$, that is, there exists a real number $\gamma>0$ such that $\langle B(t) x, x\rangle \leq-\gamma\|x\|^{2}$ and $\langle C(t) x, x\rangle \leq-\gamma\|x\|^{2}$ for all $x \in X, x \neq 0$, and $t \in[a, \infty)$.

Remark 3.3. Consider the control system

$$
\dot{x}(t)=A(t) x(t)+B u(t), \quad x(a)=x_{0},
$$

with a quadratic cost functional

$$
J[u(\cdot)]=\int_{a}^{\infty}[\langle Q x(t), x(t)\rangle+\langle R u(t), u(t)\rangle] d t
$$

where $u(t)$ and $x(t)$ take values in Hilbert spaces $U$ and $X$, where $B \in \mathscr{L}[U, X]$, and where $Q \in \mathscr{L}[X, X]$ and $R \in \mathscr{L}[U, U]$ are selfadjoint operators. 
From optimal control theory, the following Hamiltonian system is derived:

$$
\begin{gathered}
\dot{x}(t)=A(t) x(t)-B R^{-1} B p(t), \\
x(a)=x_{0}, \\
\dot{p}(t)=-A^{*}(t) p(t)-Q x(t), \\
x(\cdot) \in L^{2}([a, \infty) ; X), \quad p(\cdot) \in L^{2}([a, \infty) ; X) .
\end{gathered}
$$

This is a special case of system (1.5). Therefore, system (3.4) has a unique solution if both $B R^{-1} B$ and $Q$ are positive definite.

\section{References}

[1] Y. Hu and S. Peng, Solution of forward-backward stochastic differential equations, Probab. Theory Related Fields 103 (1995), no. 2, 273-283.

[2] J.-L. Lions, Optimal Control of Systems Governed by Partial Differential Equations, Die Grundlehren der mathematischen Wissenschaften, vol. 170, Springer, New York, 1971.

[3] S. Peng and Y. Shi, Infinite horizon forward-backward stochastic differential equations, Stochastic Process. Appl. 85 (2000), no. 1, 75-92.

Lianwen Wang: Department of Mathematics and Computer Science, College of Arts and Sciences, Central Missouri State University, Warrensburg, MO 64093, USA

E-mail address: lwang@cmsu1.cmsu.edu 




Advances in

Operations Research

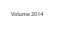

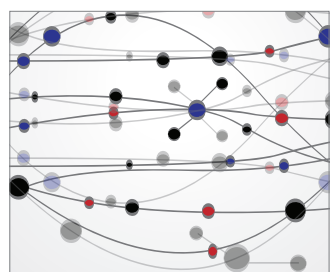

\section{The Scientific} World Journal
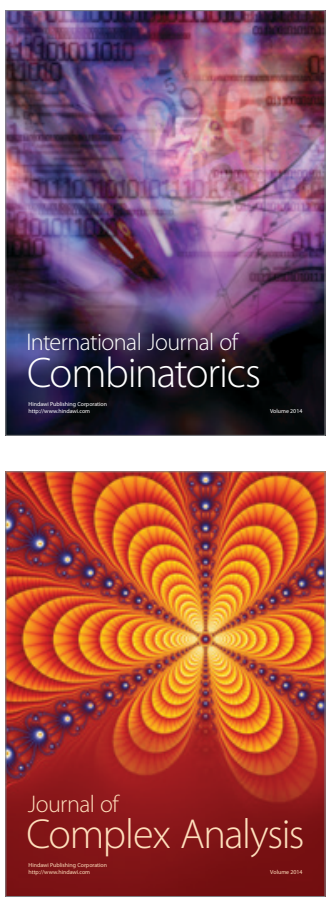

International Journal of

Mathematics and

Mathematical

Sciences
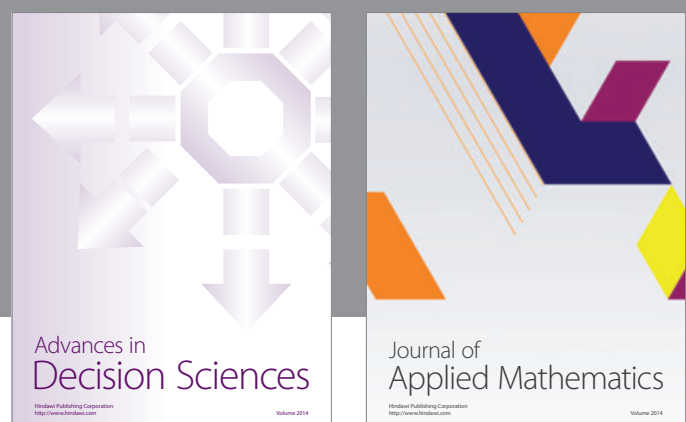

Journal of

Applied Mathematics


Submit your manuscripts at http://www.hindawi.com
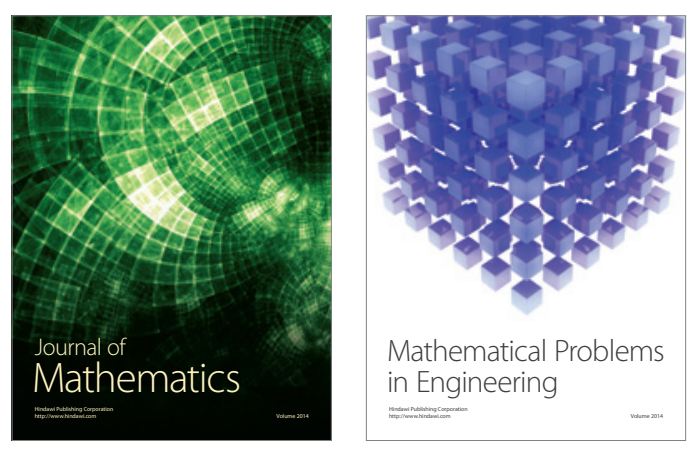

Mathematical Problems in Engineering
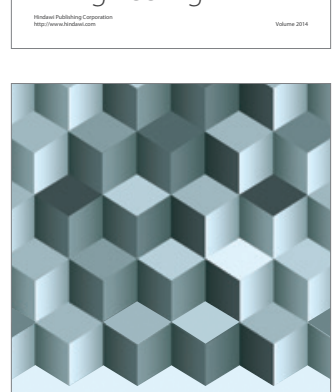

Journal of

Function Spaces
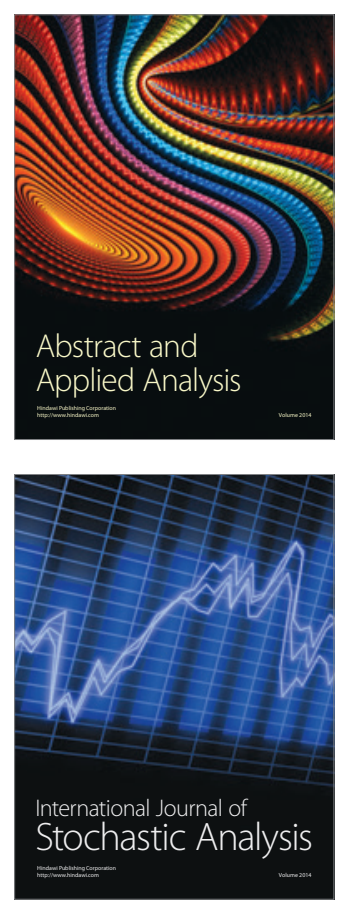

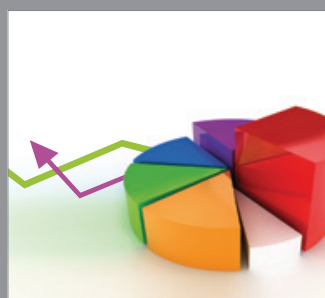

ournal of

Probability and Statistics

Promensencen
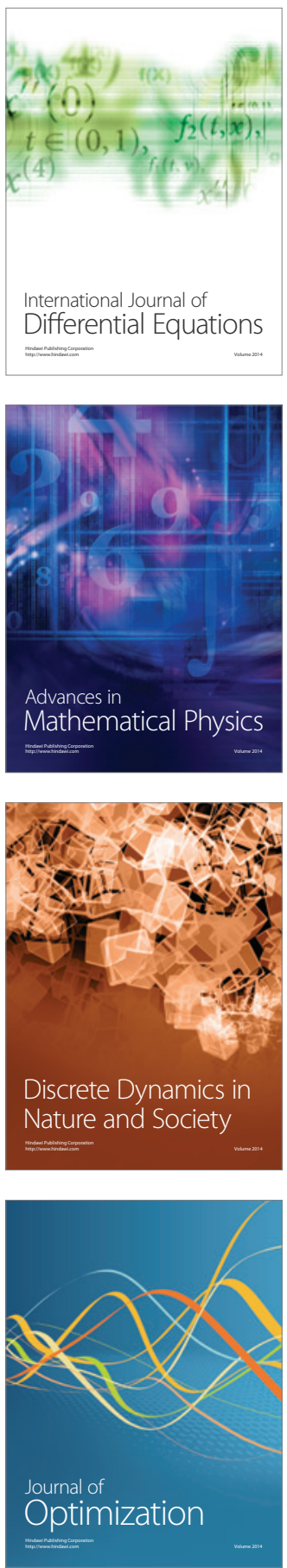